\title{
Anomalous fluctuations of nematic order in solutions of semiflexible polymers
}

\author{
Sergei A. Egorov ${ }^{1,3,4}$, Andrey Milchev ${ }^{2}$, and Kurt Binder ${ }^{3}$ \\ 1 Department of Chemistry, University of Virginia, Charlottesville, \\ VA 22901, USA [E-Mail:sae6z@cms.mail.virginia.edu] \\ 2 Institute for Physical Chemistry, Bulgarian Academia of Sciences, 1113 Sofia, Bulgaria \\ 3 Institut für Physik, Johannes Gutenberg Universität Mainz, 55099 Mainz, Germany \\ 4 Leibniz-Institut für Polymerforschung, Institut Theorie der Polymere, Hohe Str. 6, 01069 Dresden, Germany
}

\begin{abstract}
The nematic ordering in semiflexible polymers with contour length $L$ exceeding their persistence length $\ell_{p}$ is described by a confinement of the polymers in a cylinder of radius $r_{e f f}$ much larger than the radius $r_{\rho}$, expected from the respective concentration of the solution. Large scale Molecular Dynamics simulations combined with Density Functional Theory are used to locate the IsotropicNematic $(I-N)$-transition and to validate this cylindrical confinement. Anomalous fluctuations, due to chain deflections from neighboring chains in the nematic phase are proposed. Considering deflections as collective excitations in the nematically ordered phase of semiflexible polymers elucidates the origins of shortcomings in the description of the $I-N$ transition by existing theories.
\end{abstract}

Introduction.-The stiffness of semiflexible macromolecules in solutions and melts creates a tendency towards liquid-crystalline order. However, neither the precise conditions for the onset of nematic order, nor the properties of the phases are well understood. [1 4] Semiflexible polymers behave like rigid rods on the persistence length scale $\ell_{p}$, yet like random coils [5] on larger scales, if their contour length $L \gg \ell_{p}$. In solutions of semiflexible polymers good solvent conditions prevail, the effective monomer-monomer interactions being repulsive. The monomer concentration $\rho$ is then the control parameter for the onset of order. Unlike solutions of rod-like particles (e.g., the tobacco mosaic virus [6]), where translational and orientational entropy contributions compete 7], here also conformational degrees of freedom due to chain flexibility matter. This hampers the understanding of such systems [8-17]: even in the limit $\ell_{p} \gg d$ ( $d$ being the effective monomer diameter) the extension of Onsager's theory [7] for the isotropic (I) nematic $(\mathrm{N})$ transition of thin long rods is difficult. 8 Attempts [13 17] to go beyond this limit have produced contradictory results: at the concentrations of interest it no longer suffices to deal with the inter-chain interactions via the 2-nd virial coefficient only, as [7-12] for $\ell_{p} \gg d$. However, progress in the understanding of these lyotropic crystalline polymers is highly desirable in view of interesting applications (various liquid crystal devices 1, 2], emerging new types of complex soft materials such as nematic elastomers [18], nematic emulsions [19], etc.), and in the context of biological matter (the stiff cytoskeleton networks, neurofilaments within the axon [20, 21], intermediate filaments in cells [22, 23], etc.)

In the present Letter we take steps towards elucidating this important problem by means of large scale Molecular Dynamics (MD) simulations, analyzing them in terms of the "deflection length" concept. This length, $\lambda$, was originally used to describe confinement of semiflexible chains in cylindrical tubes [24 27]. We will explain why long wavelength collective fluctuations occur, causing large deflections of the polymers from their director. The observed reduction of the nematic order parameter $S$ is then stronger than predicted by Density Functional Theory (DFT), even when the DFT prediction for the location of the transition is validated by MD. 28]

Simulations of $I-N$ transitions have been attempted earlier 29 35], albeit only short chains and small simulation boxes could be handled (we disregard thereby lattice models [33 35] where the chains can order only in discrete directions and no deflection length exists). In the present work both $\ell_{p}$ and the chain length $N$ (i.e., the number of beads in chains) are widely varied, $8 \leq N \leq 128$, and large systems (up to 500000 beads) have been used. Our work has become feasible by means of very efficient codes [36, 37] on graphical processing units (GPUs). 38]

Model.-We employ the standard model [39] where beads interact along the chains with the spring potential $U^{F E N E}(r)$ while any pair of beads interact with the repulsive part of the Lennard-Jones (LJ) potential, $U^{L J}(r)$, $r$ being the distance between beads. In this model the distance between the neighboring beads along the chain is $\ell_{b}=0.970 \sigma$ (hence the contour length $L=(N-1) \ell_{b}$ ), with the LJ diameter $\sigma=d=1$ and the LJ energy $\epsilon=1$, as well as temperature $T=1$; the integration time step is $\delta t=0.01$ ( $\tau=\sqrt{m \sigma^{2} / \epsilon}=1 \mathrm{MD}$ time unit) [40]. Chain stiffness is described by the bond bending potential $U^{\text {bend }}\left(\theta_{i j k}\right)=\epsilon_{b}\left[1-\cos \left(\theta_{i j k}\right)\right]$ for $j=i+1, k=j+1$. Here $\theta_{i j k}$ is the angle between the bond vector $\vec{a}_{i}=\vec{r}_{j}-\vec{r}_{i}$ and $\vec{a}_{j}=\vec{r}_{k}-\vec{r}_{j}$. The persistence length is then simply [41] $\ell_{p} / \ell_{b}=-1 / \ln \left\langle\cos \left(\theta_{i j k}\right)\right\rangle=\epsilon_{b}$ for $\epsilon_{b} \geq 2$.

We vary $\epsilon_{b}$ from $\epsilon_{b}=8$ to $\epsilon_{b}=128$. The linear dimension $L_{b o x}$ of the cubic simulation box (with periodic boundary conditions) is chosen large enough so that even fully extended chains still fit in. Thus, with $\mathcal{N}$ chains altogether, the monomer concentration $\rho=\mathcal{N} N / L_{\text {box }}^{3}$. System trajectories are computed with the velocityVerlet algorithm, applying as usual a Langevin 39] thermostat. Pressure $P$ is computed using the Virial theorem, and the order parameter $S$ is the largest eigenvalue of the tensor $Q_{\alpha \beta}$ which describes the average orientation of the unit vectors along bonds in the system. [42]

Fig. 17 shows a typical configuration in the nematic phase. Although there the value of $S$ is large $(S \approx$ 

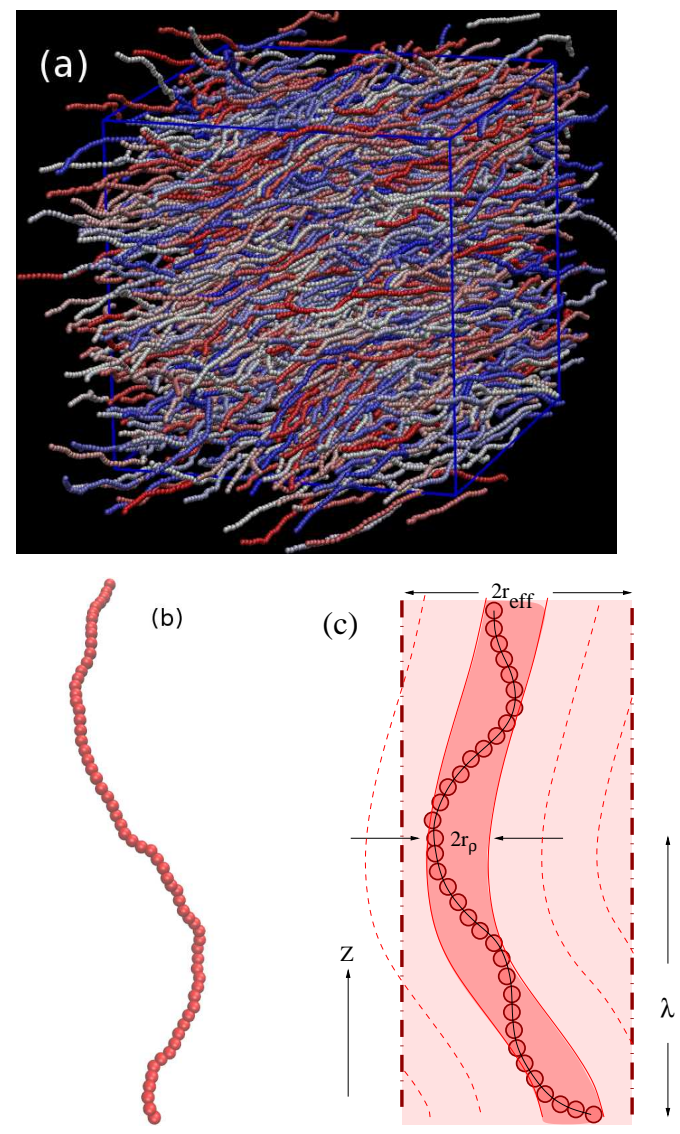

FIG. 1: (a) Snapshot of a system of semiflexible polymers with length $N=32$, stiffness $\epsilon_{b}=100$, at concentration $\rho=0.6$ (deep in the nematic phase). (b) Typical conformation of a semiflexible polymer in the nematic phase $\left(N=64, \epsilon_{b}=\right.$ 16, $\rho=0.4)$. (c) Schematic description of nematic order: each chain has its own cylindrical (bent) tube of diameter $2 r_{\rho}$, defined such that it contains only monomers from the considered chain. The tube is placed inside a straight wider cylinder of diameter $2 r_{e f f}$ (see text). The definition of the deflection length $\lambda$ is indicated.

$0.9)$, considerable bending of the wormlike chains is observed. On the molecular scale, the character of this phase differs considerably from a nematic formed by rod-like molecules. Long wavelength excitations (deflections of chain orientation around the common director) are clearly seen from typical configurations of individual chains as the simulation snapshots prove (Fig. 1b). These observations suggest a more comprehensive coarsegrained picture of nematic order in solutions of semiflexible polymers (Fig. 1 ) that we explain below.

Equation of state and order parameter.-Typical data for pressure (Fig. 2a) versus concentration reveal qualitative agreement between MD and DFT. Of course, the latter cannot use the continuous potentials used by MD, but rather is based on extensions of a tangent hard-sphere chain model [17, 42], and different choices of the equation of state within the DFT framework yield slightly different results (see Supplementary Information). Thus, perfect quantitative agreement between the DFT prediction for the location of the $I-N$ transition and the simulation cannot be expected. Interestingly, Fig. $2 \mathrm{~b}$ reveals a similar trend as the Khokhlov-Semenov-Odijk-Chen [8 12 ] theory, when we plot the volume fraction $\rho_{t r} \pi / 4$ at the transition multiplied by $\ell_{p} / d$ versus $L / \ell_{p}$. However, unlike usually assumed [4, 8 12, 30], this does not yield a universal master curve, but rather a decrease of $\rho_{t r}$ (at fixed $\left.L / \ell_{p}\right)$ with increasing ratio $d / \ell_{p}$ takes place. In fact, this finding helps understand the origin of discrepancies between theories [8 [17] and experiments [4, 43, 44] where a fit of all systems to a universal master curve was assumed [4, 30].

However, most interesting is the qualitative discrepancy between MD and DFT with respect to the density dependence of the order parameter $S$ (Fig. 3), whereby the DFT result approaches saturation much faster than according to MD, that is, DFT significantly overestimates the degree of ordering in the nematic phase. We attribute this fact to the neglect of long wavelength fluctuations in the nematic phase, reflecting the mean-field character of the DFT. The situation is analogous to the case of the molecular field approximation (MFA) for an isotropic Heisenberg ferromagnet: the MFA also does not allow for effects due to magnons. Both in this case and at the $I-N$ transition, a continuous symmetry is broken, but for semiflexible polymers the situation is special since an additional lengthscale (the deflection length) matters.

Deflection length and cylindrical confinement.-Unlike nematic order of rigid rods, the local order parameter $S_{i}$ along the contour of an individual chain is non-uniform (Fig. 4a), and can be described by

$$
S_{\infty}-S(i) \propto \exp \left(-i \ell_{b} / \lambda\right)
$$

$S_{\infty}$ being the order parameter in the center of a chain (for $L \rightarrow \infty$ ), and $\lambda$ can be taken as a definition of the deflection length [10-12]. Alternatively, we can measure the mean-square monomer displacement $\left\langle\left(\vec{r}_{i, \perp}-\vec{r}_{j, \perp}\right)^{2}\right\rangle$ in the direction perpendicular to the end-to-end vector $\vec{r}_{N}-\vec{r}_{1}$ as function of the bead index (Fig. 4 $4 \mathrm{~b}$, inset). In the nematic phase this displacement increases linearly with $s=j-i$ and reaches a flat maximum (of height $\left.r_{\text {eff }}^{2}\right)$ at distance $\lambda$ along the contour. The deflection length is normally 24 27] defined for a semiflexible polymer confined in a cylinder of radius $r_{e f f}$.

Considering the initial growth of the mean-squared angle with the distance $s$ along the contour, $\left\langle\theta^{2}(s)\right\rangle=$ $2 s \ell_{b} / \ell_{p}$, and equating this to $r_{\text {eff }}^{2} / \lambda^{2}$ for $s \ell_{b}=\lambda$, one concludes that $\lambda=\left(\ell_{p} r_{\text {eff }}^{2}\right)^{1 / 3}$ and $1-S \approx 3 / 2\left\langle\theta^{2}\right\rangle \approx$ $3 / 2\left(r_{e f f} / \ell_{p}\right)^{2 / 3}$. Since the average projection of each bond along the cylinder axis is $\ell_{b}\langle\cos \theta\rangle \approx \ell_{b}\left(1-\left\langle\theta^{2}\right\rangle / 2\right)$, the reduction of the mean-squared end-to-end distance becomes $1-\left\langle R_{e}^{2}\right\rangle^{1 / 2} / L \approx\left\langle\theta^{2}\right\rangle / 2=\left(\frac{r_{e f f}}{\ell_{p}}\right)^{2 / 3} / 2$. These scaling arguments can be made more precise to yield, 25 27] for $L / \ell_{p} \gg 1$, 

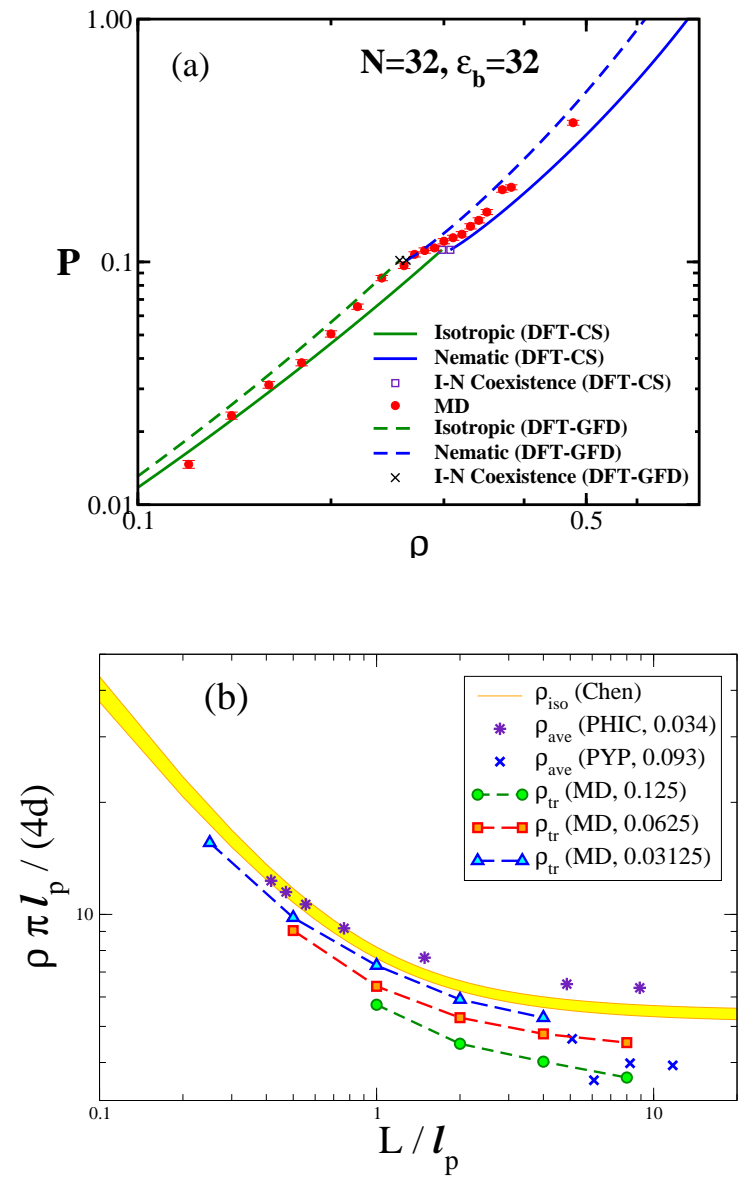

FIG. 2: Pressure vs concentration for the case $N=32, \epsilon_{b}=$ 32 , according to MD (full dots) and two versions of density functional theory, DFT-CS (solid lines) and DFT-GFD (broken lines) 28]. The $I-N$ transition in the simulation is rounded by finite-size effects. DFT predictions for $I-N$ coexistence are indicated by squares (DFT-CS) and crosses (DFT-GFD). (b) Scaled volume fraction $\rho \pi \ell_{p} /(4 d)$ at the transition plotted versus $L / \ell_{p}$ according to MD, theory [12] and typical experiments [43, 44]. The shaded stripe indicates the I-N coexistence region, $\rho_{i}<\rho<\rho_{n}$, as predicted by Chen [12]. MD does not resolve $\rho_{i}, \rho_{n}$, rather $\rho_{t r}$ is the position of the maximum slope of the $S$ vs $\rho$ curve (see Fig. 3). For the experiments, namely poly(hexyl isocyanate) (PHIC) in toluene [4] and poly(yne)-platinum (PYP) in trichloroethylene [43] $\rho_{\text {ave }}=\left(\rho_{i}+\rho_{n}\right) / 2$ was taken as the transition density. The numbers in the brackets in the legend indicate $d / \ell_{p}$.

$$
1-S=3\left(1-\frac{\sqrt{\left\langle R_{e}^{2}\right\rangle}}{L}\right)=0.51\left(\frac{2 r_{e f f}}{\ell_{p}}\right)^{2 / 3}=3 \frac{\lambda}{\ell_{p}} .
$$

We now suggest that nematic order of semiflexible polymers can be essentially understood in terms of cylindrical confinement as a collective effect of the neighboring chains of the considered chain (Fig. 15). These cylinders in Fig. 11 must not be confused with the tubes due to entanglements in solutions of semiflexible polymers, controlling the viscoelastic dynamics in the isotropic

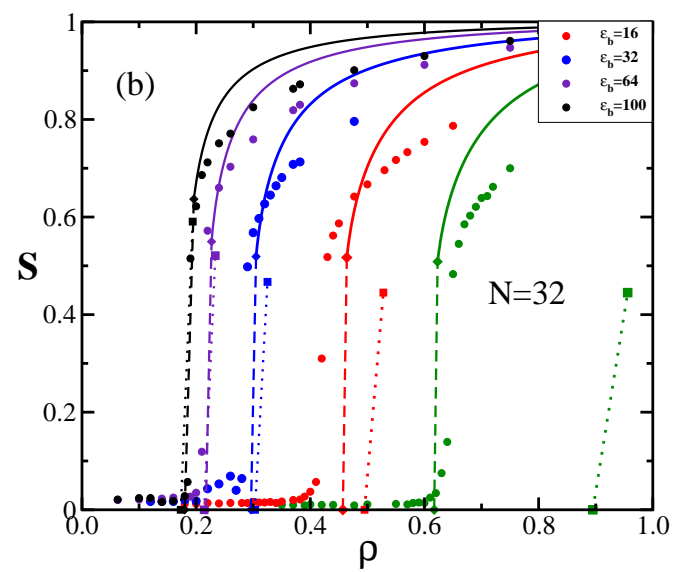

FIG. 3: Nematic order parameter $S$ from MD (filled circles) vs concentration $\rho$ for $N=32$ and various choices of $\epsilon_{b}$, as indicated. Full curves denote corresponding predictions of DFT-CS. 28] $I-N$ coexistence is indicated by diamonds and broken straight lines (lever rule). Corresponding predictions from Chen [12] are shown by squares and dotted lines.

phase [45-48]. Fig. 4b shows how both $\lambda$ and $r_{\text {eff }}$ can be extracted from the data. Choosing different values of $N$ and $\epsilon_{b}$, we can also test the left part of Eq. (2), see Fig. 5. For the regime where Eq. (2) should hold, namely $L / \ell_{p} \gg 1$ and $\left\langle\theta^{2}\right\rangle \ll 1$, i.e. $1-S \leq 0.2$, we get very good agreement with no adjustable parameters whatsoever. For $L / \ell_{p} \leq 1$, the data display curvature and bend upwards away from a straight line. This is expected, of course, since for $L / \ell_{p}<1$ the end-to-end distance of such rather stiff "flexible rods" cannot decrease much. Disordering of the nematic phase then occurs predominantly due to misorientation of the flexible rods relative to the director as a whole. Remarkably, different choices of $L$ and $\ell_{p}$ in the representation of Fig. 囵yield a set of master curves depending on the single parameter $L / \ell_{p}$ only. However, different scaled concentrations $\rho \ell_{p} / d$ for a given $L / \ell_{p}$ do not coincide on the same point of the master curve, but differ systematically. Using the result $1-S=3\left(\lambda / \ell_{p}\right)$, we obtain alternative estimates for $\lambda$. For the cases shown in Fig. [3a, we thus find for $\epsilon_{b}=8,16$, and 32 the values $\lambda=1.36,2.14$, and 4.7 , respectively. These estimates are systematically somewhat smaller than those extracted from Fig. 4a via Eq. (1), but exhibit a similar trend.

This analysis in terms of cylindrical confinement does not mean that the nearest neighbors of a chain enclose it in a cylinder of radius $r_{\text {eff }}$ (Fig. 15), rather this cylinder is shared by many chains. This is readily seen when we compute a radius of a cylinder from $\rho$ via $r_{\rho}=\left[N /\left(\pi \rho\left\langle R_{e}^{2}\right\rangle^{1 / 2}\right)\right]^{1 / 2}$, i.e., a cylinder containing the monomers of one chain only (and solvent particles). For concentrated solutions $r_{\rho}$ is comparable to $\sigma$, of course, see Fig. 4b, while $r_{e f f}$ extracted from Eq. (2) is much larger (it increases proportional to $\ell_{p}$ ). This description implies Fig. 1r, i.e., each chain is confined in a tube of radius $r_{\rho}$, but this tube as a whole is like a 

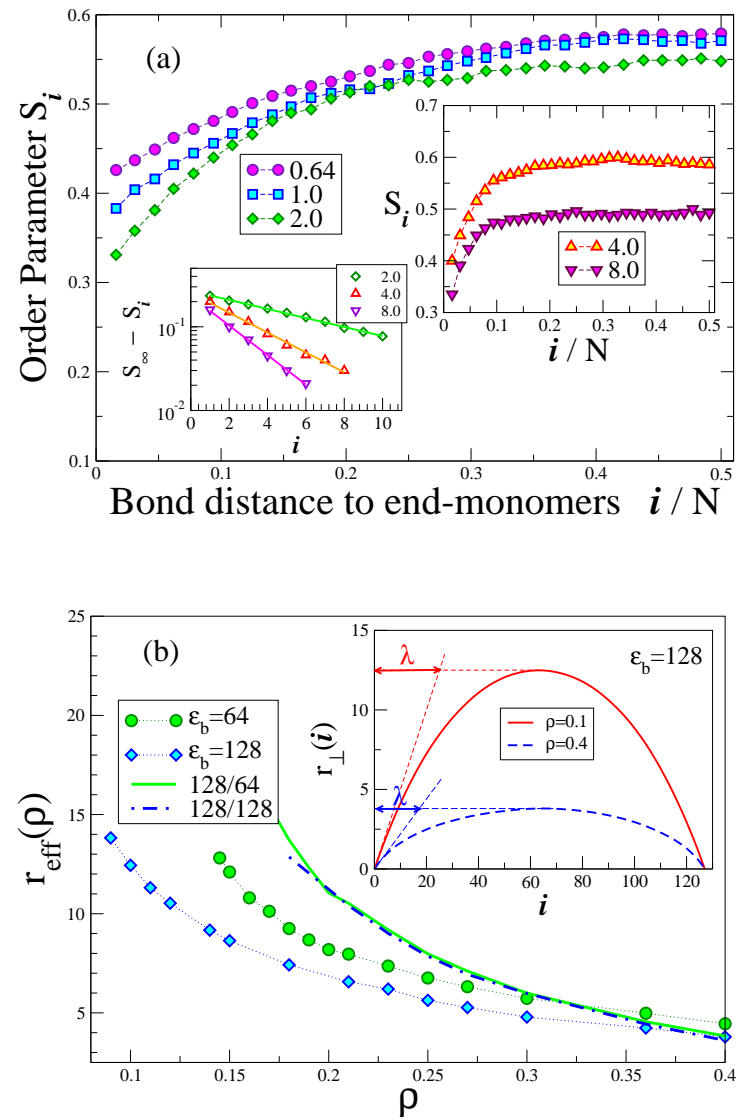

FIG. 4: (a) Local order parameter $S_{i}$ (referring to bond vector $\vec{a}_{i}$ ) plotted vs $i / N$ and averaged over all equivalent bonds in the system for the case $N=64$ and several choices of $N / \epsilon_{b}$, as indicated. The left inset shows a semi-log plot of $S_{\infty}-S_{i}$ vs $i$ so as to demonstrate Eq. 2 From the slope the deflection length $\lambda$ is extracted as $\lambda / \ell_{p}=2.36,3.65$, and 8.2 for $\epsilon_{b}=8,16$, and 32, respectively. (b) Variation of the confinement radius $r_{\text {eff }}$ with concentration $\rho$ for semiflexible chains with $N=128$ and two degrees of stiffness $\epsilon_{b}=64$ and 128, computed from Eq. (2) and from the maximum of $\left\langle\left(r_{i, \perp}-r_{j, \perp}\right)^{2}\right\rangle$. The inset shows the mean-squared displacement of consecutive beads, $\left\langle\left(r_{i, \perp}-r_{j, \perp}\right)^{2}\right\rangle$, perpendicular to the respective end-to-end vector $\vec{R}_{e}$, averaged over all chains in the nematic phase for $\epsilon_{b}=128$ and two densities.

wormlike chain, making excursions of order of $r_{\text {eff }}$ on a length scale $\lambda$ along the cylinder axis. Since the cylinder of radius $r_{\text {eff }}$ contains a bundle of chains (which may be twisted around each other, a feature missed in our two-dimensional cartoon), it is clear that the deflections of these chains sharing one cylinder are coherent collective excitations, because the tubes of radius $r_{\rho}$ must be essentially space-filling. Of course, for a semidilute solution $r_{\rho}$ may exceed $\sigma$ considerably, and then the chains have additional bending degrees of motion within their individual tubes as indicated qualitatively in Fig. 11.

Conclusions. - In summary, we have shown that the nematic phase of semiflexible polymers exhibits collective deflection modes on length scale $\lambda$ of amplitude $r_{\text {eff }}$

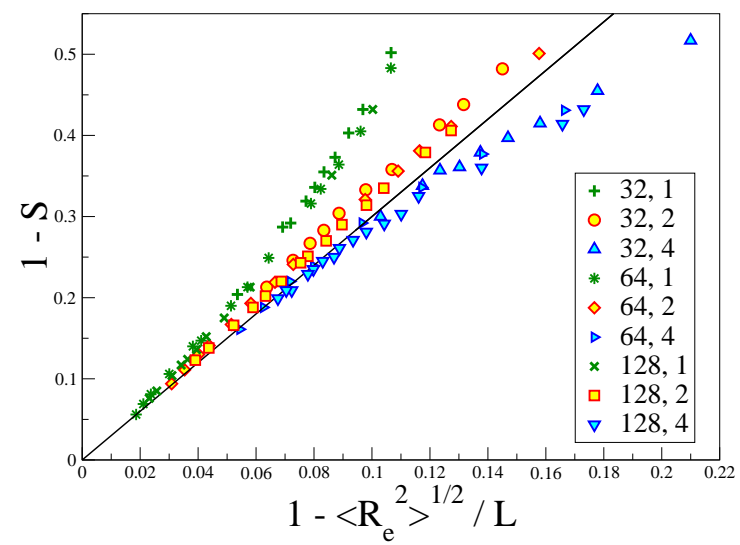

FIG. 5: Plot of $1-S$ vs the relative reduction $1-\left\langle R_{e}^{2}\right\rangle^{1 / 2} / L$ of the end-to-end distance for three choices of $N=32,64$, and 128 , and three choices of the $N / \epsilon_{b}=1,2$, and 4 , as indicated. Different points with the same symbol refer to different choices of the density $\rho$. The fully stretched chain would be the origin of the plot whereas the straight line shows Eq. (2). Rigid rods would correspond to the ordinate axis here.

perpendicular to the director, if $L / \ell_{p} \gg 1$, and both $\lambda$ and $r_{\text {eff }}$ can be directly predicted from the order parameter $S$ (Eq. (21)). We feel that the picture of nematic order of semiflexible polymers developed here has also important implications for both linear and nonlinear elastic response of such systems, and corresponding experiments testing our ideas would be very welcome. So far the deflection length has only been measured for a semiflexible chain in a nematic solvent 49]. There one might need to consider defects such as hairpins in the structure (which have been occasionally detected in our simulations). Note that in nematics formed from rigid rods each rod is confined in a cylinder of radius $r_{\rho}$ while the length scales $\lambda$ and $r_{\text {eff }}$ do not exist! For the interpretation of the experiments, the version of DFT that we have used and validated here could be very useful, since it can be worked out for a much wider parameter range compared to MD. Our study clearly shows limitations of the previous theories of the $I-N$ transition of semiflexible polymers, see, e.g., Fig. $2 \mathrm{~b}$, and should provide a better understanding of experiments. It would be very interesting to study the corresponding static and dynamic collective structure factors, but this is beyond our scope here. It remains a challenge to extend the analytic theories [8-12, 26] to self-consistently predict the length scales $\lambda$ and $r_{\text {eff }}$ from the molecular parameters $\ell_{p}, d$ and $L$ and the polymer density.

\section{Acknowledgements}

S.A.E. acknowledges financial support from the Alexander von Humboldt Foundation. A.M. thanks for partial support under the grant No BI314/24. We are particularly indebted to Dr. P. Virnau for his help and advice with GPU computing. Parts of this research were conducted using the supercomputer Mogon and/or advisory services offered by Johannes Gutenberg Univer- 
sity Mainz (www.hpc.uni-mainz.de), which is a member of the AHRP and the Gauss Alliance e.V. The authors gratefully acknowledge the computing time granted on the supercomputer Mogon at Johannes Gutenberg University Mainz (www.hpc.uni-mainz.de).
[1] A. Ciferri (ed.), Liquid Crystallinity in Polymers: Principles and Fundamental Properties (VCH Publishers: New York 1983).

[2] A. M. Donald, A. H. Windle, and S. Hanna, Liquid Crystalline Polymers (Cambridge University Press: Cambridge 2006).

[3] A. Y. Grosberg and A. R. Khokhlov, Adv. Polym. Sci. 41, 53 (1981).

[4] T. Sato and A. Teramoto, Adv. Polym. Sci. 126, 85 (1996).

[5] P. G. de Gennes, Scaling Concepts in Polymer Physics (Cornell University Press: Ithaca 1979).

[6] S. Fraden, G. Maret, D. L. D. Caspar, and R. B. Meyer, Phys. Rev. Lett. 63, 2068 (1989).

[7] L. Onsager, Ann. N. Y. Acad. Sci. 51, 627 (1949).

[8] A. R. Khokhlov and A. N. Semenov, Physica A 108, 546 (1981).

[9] A. R. Khokhlov and A. N. Semenov, Physica A 112, 605 (1982).

[10] T. Odijk, Macromolecules 26, 197 (1985).

[11] T. Odijk, Macromolecules 19, 2313 (1986).

[12] Z. Y. Chen, Macromolecules 26, 3419 (1993).

[13] R. Hentschke, Macromolecules 23, 1192 (1990).

[14] D. B. DuPré and S.-J. Yang, J. Chem. Phys. 94, 7466 (1991).

[15] T. Sato and A. Teramoto, Mol. Cryst. Liq. Cryst. 178, 143 (1990).

[16] T. Sato and A. Teramoto, Acta Polymerica 45, 399 (1994).

[17] H. Fynewever and A. Yethiraj, J. Chem. Phys. 108, 1636 (1998).

[18] H. Finkelmann, E. Nishikawa, G. G. Pereira, and M. Warner, Phys. Rev. Lett. 87, 015501 (2001).

[19] P. Poulin, H. Stark, T. C. Lubensky, and D. A. Weitz, Science 275, 1770 (1997).

[20] R. A. Aldoroty, N. B. Garty, and E. W. April, Biophys. J. 51, 371 (1987).

[21] N. Hirokawa, M. A. Gliksman, and M. B. Willard, J. Cell Biol. 98, 1523 (2015).

[22] S. Koster, D. A. Weitz, R. D. Goldman, U. Aebi, and H. Herrmann, Curr. Opinion Cell Biol. 32, 82 (2015).

[23] F. Huber, A. Boire, M. P. Lopez, and G. H. Koenderink, Curr. Opinion Cell Biol. 32, 39 (2015).

[24] T. Odijk, Macromolecules 16, 1340 (1983).

[25] Y. Z. Yang, T. W. Burkhardt, and G. Gompper, Phys. Rev. E 76, 011804 (2007).

[26] J. Z. Y. Chen, Macromolecules 46, 9837 (2013).
[27] J. Z. Y. Chen, Prog. Polym. Sci. xx, in press (2015).

[28] cf. Supplementary Information for the technical details regarding DFT.

[29] M. R. Wilson and M. P. Allen, Mol. Phys. 80, 277 (1993).

[30] M. Dijkstra and D. Frenkel, Phys. Rev. E 51, 5891 (1995).

[31] F. A. Escobedo and J. J. dePablo, J. Chem. Phys. 106, 9858 (1997).

[32] T. van Westen, B. Oyarzun, T. J. H. Vlugt, and J. Gross, J. Chem. Phys. 139, 034505 (2013).

[33] H. Weber, W. Paul, and K. Binder, Phys. Rev. E 59, 2168 (1999).

[34] V. A. Ivanov, M. R. Stukan, M. Müller, W. Paul, and K. Binder, J. Chem. Phys. 118, 10333 (2003).

[35] V. A. Ivanov, E. A. An, L. A. Spirin, M. R. Stukan, M. Müller, W. Paul, and K. Binder, Phys. Rev. E 76, 026702 (2007).

[36] J. Anderson, C. Lorenz, and A. Travesset, J. Comput. Phys. 227, 5342 (2008).

[37] J. Glaser, T. D. Nguyen, J. A. Anderson, P. Liu, F. Spiga, J. A. Millan, D. C. Morse, and S. C. Glotzer, Comp. Phys. Comm. 192, 97 (2015).

[38] cf. Supplementary Information for the technical details regarding the GPUs.

[39] K. Kremer and G. S. Grest, J. Chem. Phys. 92, 5057 (1990).

[40] cf. Supplementary Information for the technical details regarding the model and the MD simulations.

[41] H.-P. Hsu, W. Paul, and K. Binder, Macromolecules 43, 3094 (2010).

[42] S. A. Egorov, A. Milchev, P. Virnau, and K. Binder, xx $\mathbf{x x}$, to be submitted (2016).

[43] A. Abe, N. Kimura, and S. Tabata, Macromolecules 24, 6238 (1991).

[44] T. Itou and A. Teramoto, Macromolecules 21, 2225 (1988).

[45] D. C. Morse, Phys. Rev. E 58, 1237 (1998).

[46] S. Ramanathan and D. C. Morse, Phys. Rev. E 76, 010501 (2007).

[47] H. Hinsch, J. Wilhelm, and E. Frey, Eur. Phys. J. E 24, 35 (2007).

[48] J. Glaser and K. Kroy, Phys. Rev. E 84, 051801 (2011).

[49] Z. Dogic, J. Zhang, A. W. C. Lau, H. ArandaEspinoza, P. Dalhaimer, D. E. Discher, P. A. Janmey, R. D. Kamien, T. C. Lubensky, and A. G. Yodh, Phys. Rev. Lett. 92, 125503 (2004). 